\title{
A Novel Technique for Estimating Frequency Offset in Orthogonal Frequency Division Multiplexing
}

\author{
Mustafa Altaha and Humor Hwang ${ }^{+}$ \\ Department of Information and Communications Engineering, Myongji University, Yongin, Korea.
}

\begin{abstract}
Orthogonal Frequency Division Multiplexing (OFDM) is broadly considered as an effective approach for current and future high-speed wireless communication. However, one of the main drawbacks of the OFDM is sensitive to carrier frequency offset (CFO). The CFO causes interference among the multiplicity of carriers in the OFDM signal. Thus, CFO must be estimated and compensated to minimize adverse effects of inter-carrier interference on the signal and maintain orthogonality. Moose technique shows a novel approach to estimate $\mathrm{CFO}$ values that less than 0.5 using a repeated data symbol. It was successfully able to estimate accurate CFO values using a maximum likelihood estimation algorithm. Moose also describes how to estimate CFO values greater than 0.5 using shorter training symbols, but the accuracy of the CFO values have decresed. In this paper, we present a novel technique to estimate CFO values that are greater than 0.5 while maintaining their accuracy level. Our technique is used to estimate large range of CFO values using shorter training symbols and then determine a numerical multiplicand by a step function. Based on a numerical multiplicand, the CFO values will be reduced in order that the maximum likelihood estimation algorithm can be applied because the CFO values became less than 0.5. Consequently, the estimation performance is increased and the estimation range becomes wider. The analytical analysis shows better mean square error for our technique compared to Moose technique.
\end{abstract}

Keywords: orthogonal frequency division multiplexing (OFDM), carrier frequency offset (CFO), maximum likelihood estimation (MLE).

\section{Introduction}

One of the principal advantages of the OFDM is its robust against multi-path channel which can cause inter-symbol interference (ISI) and inter-carrier interference (ICI). However, this is prevented in OFDM by the insertion of a cyclic prefix between successive OFDM symbols [1].

However, one of the main disadvantages of OFDM systems is sensitivity against carrier frequency offset (CFO), which causes inter-carrier interference. The sensitivity of the OFDM to the CFO in single carrier systems is a critical issue [2]. In general, the CFO is defined as the difference between the nominal frequency and actual output frequency. In OFDM, the uncertainty in carrier frequency due to a difference in the frequencies of the local oscillators in the transmitter and the receiver sides gives rise to a shift in the frequency domain. This shift is also referred as frequency offset. It can also be caused due to Doppler shift in the channel. The demodulation of a signal with offset in the carrier frequency can cause large bit error rate and may degrade the performance of a symbol synchronizer. Therefore, it is important to estimate the frequency offset and minimize or eliminate its impact [3]. However, several techniques have proposed to estimate the CFO in time domain and frequency domain.

Moose [4] proposed a technique for a maximum likelihood estimation (MLE) of frequency offset using the discrete Fourier transform (DFT) values of a repeated data symbol. In this technique, the accuracy that required by the frequency offset correction depends on how much residual offset can be tolerated. The acquisition range of the technique is $\pm 1 / 2$ of the intercarrier spacing for the repeated symbol. Beek,

\footnotetext{
+ Corresponding author. Tel.: +8210-5326-0590

E-mail address: hmhwang@mju.ac.kr
} 
Sandell and Borjesson [5] proposed the joint maximum likelihood estimator of time and frequency offset in ODFM systems. The estimation uses the redundant information contained within the cyclic prefix. Zhou et al. [6] presented two maximum likelihood CFO estimation schemes, one in frequency domain and another in time domain, both are under Doppler fading. Classen and Meyr [7] introduced a method to find both the symbol timing and carrier frequency offset. Pilot tones can be inserted in the frequency domain and transmitted in every OFDM symbol for the CFO tracking. After estimating CFO from pilot tones in the frequency domain, the signal is compensated with the estimated $\mathrm{CFO}$ in the time domain.

In this paper, we propose a novel technique for estimating CFO in OFDM systems. Our proposed technique increases the range of estimation with preserving the estimation accuracy using shorter training symbols and applying a step function to reduce the frequency offset.

The rest of this paper is organized as follows. In Section 2, we introduce a background of the CFO in OFDM systems. Section 3, we present our proposed technique. The performance analysis is conducted in Section 4. Finally, we discuss our conclusions and future work in Section 5.

\section{Background}

\subsection{CFO effect}

In OFDM transmission scheme a wideband channel is divided into $\mathrm{N}$ orthogonal narrow-band subchannels. N-point IDFT and DFT are used to implement OFDM modulation and demodulation, The transmitter maps the message bits into a sequence of quadrature amplitude modulation (QAM). Symbols which are subsequently converted into an $\mathrm{N}$ parallel bit stream. Each of $\mathrm{N}$ symbols from the serial-to-parallel conversion is modulated in different sub-carriers. An OFDM transmission symbol is given by the $\mathrm{N}$ point complex modulation sequence.

$$
X(n)=\frac{1}{N} \sum_{k=0}^{N-1} X(k) e^{2 \pi j n k / N}, \quad n=0,1, \ldots, N-1
$$

where $X(k)$ are the modulation symbols by the transmitter side.Then, cyclic prefix to preserve the sub carrier from intersymbol interference is added. The cyclic prefix aids in removing the effect of the channel (a delay version of the signal). After passing through a bandpass channel, the complex envelope of the received sequence can be expressed as follows:

$$
\mathrm{Y}(\mathrm{n})=\frac{1}{\mathrm{~N}} \sum_{\mathrm{k}=0}^{\mathrm{N}-1} \mathrm{X}(\mathrm{k}) \mathrm{H}(\mathrm{k}) \mathrm{e}^{2 \pi \mathrm{j}(\mathrm{n}+\epsilon) \mathrm{k} / \mathrm{N}}+\mathrm{W}(\mathrm{n})
$$

where $\mathrm{Y}(\mathrm{n})$ is the received signal under the presence of $\mathrm{CFO}, \mathrm{H}(\mathrm{k})$ is the channel impulse response, $\mathrm{W}(\mathrm{n})$ is the additive white Gaussian noise and $\epsilon$ is the normalize carrier frequency offset. The received signal goes through a serial to parallel converter and cyclic prefix is removed. After the cyclic prefix is removed, the signals are passed through an N-point DFT to convert the time domain signal to the frequency domain as illustrated in Eq. (3).

$$
Y(k)=\sum_{n=0}^{N-1} Y(n) e^{-j 2 \pi k n / N}
$$

Because of the frequency offset, the output of the discrete Fourier transform is formed from the first $\mathrm{N}$ samples of the output as shown in Eq. (4).

$$
\mathrm{Y}(\mathrm{k})=\frac{\sin (\pi \epsilon)}{\mathrm{N} \sin (\pi \epsilon / \mathrm{N})} \mathrm{e}^{-\mathrm{j} 2 \pi \epsilon\left(\mathrm{N}-\frac{1}{\mathrm{~N}}\right)} \mathrm{X}(\mathrm{k}) \mathrm{H}(\mathrm{k})+\mathrm{I}(\mathrm{k})+\mathrm{W}(\mathrm{k})
$$


The first part of Eq. (4) is the modulated value has modified by the channel transfer function. This part experiences an amplitude reduction and phase shift due to the frequency offset. The second part is the ICI that is caused by the frequency offset. However,

$$
\mathrm{I}(\mathrm{k})=\sum_{\substack{\mathrm{l}=0 \\ \mathrm{l} \neq \mathrm{k}}}^{\mathrm{N}-1} \mathrm{X}(\mathrm{k}) \mathrm{H}(\mathrm{k}) \frac{\sin (\pi \epsilon)}{\mathrm{N} \sin \left(\frac{\pi(\mathrm{l}-\mathrm{k}+\epsilon)}{\mathrm{N}}\right)} \mathrm{e}^{\mathrm{j} 2 \pi \epsilon(\mathrm{N}-1) / \mathrm{N}} \mathrm{e}^{-\mathrm{j} 2 \pi(\mathrm{l}-\mathrm{k}) / \mathrm{N}}
$$

In Eq. (4), there is existence for the frequency offset, in the other side, if there is no frequency offset $(\epsilon=0)$, the received signal must be as follows:

$$
\mathrm{Y}(\mathrm{k})=\frac{1}{\mathrm{~N}} \mathrm{X}(\mathrm{k}) \mathrm{H}(\mathrm{k})
$$

\subsection{CFO estimation techniques}

Moose [4] presented an algorithm to estimate the carrier frequency offset by taking the maximum likelihood estimate using the values of a repeated data symbol. In this technique, if two identical training symbols are transmitted consecutively, offset of $\epsilon$ are related with each other as follows.

$$
\mathrm{Y}_{2}(\mathrm{k})=\mathrm{Y}_{1}(\mathrm{k}) \mathrm{e}^{\mathrm{j} 2 \pi \epsilon}
$$

the CFO can be estimated as follows:

$$
\hat{\varepsilon}=\frac{1}{2 \pi} \tan ^{-1}\left\{\sum_{\mathrm{K}=1}^{\mathrm{M}} \operatorname{Im}\left[\mathrm{Y}_{2 \mathrm{k}} \mathrm{Y}_{1 \mathrm{k}}^{*}\right] / \sum_{\mathrm{K}=1}^{\mathrm{M}} \operatorname{Re}\left[\mathrm{Y}_{2 \mathrm{k}} \mathrm{Y}_{1 \mathrm{k}}^{*}\right]\right\}
$$

where $\mathrm{M}$ is the number of repeated symbols, $\mathrm{Y}_{1 \mathrm{k}}^{*}$ is the conjugate of the first symbol, and $\mathrm{Y}_{2 \mathrm{k}}$ is the second symbol. The acquisition range of Eq. (8) is $|\epsilon| \leq 0.5$ which is the limit of this algorithm.

The range of carrier frequency offset estimation can be increased by reducing the distance between two blocks of samples for correlation [8]. This is made possible by using training symbols that are repetitive with some shorter period. Let D be an integer that represents the ratio of the OFDM symbol length to the length of a repetitive pattern.

$$
\hat{\varepsilon}=\frac{D}{2 \pi} \arg \left\{\sum_{n=0}^{\frac{N}{D}-1} y_{1}^{*}[n] y_{1}\left[n+\frac{N}{D}\right]\right\}
$$

As the estimation range of CFO increase, the means square error (MSE) performance decrease. By taking the average of the estimates with the repetitive patterns of the shorter period as illustrated in Eq. (10), the MSE performance can be improved without reducing the estimation range of CFO.

$$
\widehat{\varepsilon}=\frac{D}{2 \pi} \arg \left\{\sum_{m=0}^{\mathrm{D}-2} \sum_{\mathrm{n}=0}^{\mathrm{N}} \mathrm{y}_{1}^{*}\left[\mathrm{n}+\frac{\mathrm{mN}}{\mathrm{D}}\right] \mathrm{y}_{1}\left[\mathrm{n}+\frac{(\mathrm{m}+1) \mathrm{N}}{\mathrm{D}}\right]\right\}
$$

\section{Proposed Technique}

Shorter training symbols causes increasing in the range of estimation. For example, shortening the training symbols by a factor of two will double the range of CFO estimation. However, the estimation gets 
worse as the symbols get shorter because fewer samples will be available for averaging. In this paper, we use shorter training symbols to estimate large range of CFO as illustrated in Eq. (10). Step function is applied to determine numerical multiplicand of 0.4 as we used. Based on that numerical multiplicand, we reduce the frequency offset to apply the MLE algorithm that work for the frequency offset less than 0.5 .

The step function is built for numerical multiplicand of 0.4 based on the frequency offset estimation from Eq. (9):

$$
\mathrm{S}(\widehat{\varepsilon})=\sum_{\mathrm{n}=0}^{\mathrm{N}} \mathrm{u}(\hat{\varepsilon}-(0.4+0.4 \mathrm{n}))
$$

the summation of the unit step function builds a numerical multiplicand of 0.4 as illustrated in Eq. (12):

$$
S(\widehat{\varepsilon})=\left\{\begin{array}{rr}
0 & 0 \leq \hat{\varepsilon}<0.4 \\
1 & 0.4 \leq \hat{\varepsilon}<0.8 \\
2 & 0.8 \leq \widehat{\varepsilon}<1.2 \\
3 & 1.2 \leq \widehat{\varepsilon}<1.6 \\
\ldots &
\end{array}\right.
$$

$S(\hat{\varepsilon})$ is an integer number. The purpose of the numerical multiplicand is to reduce the frequency offset by $S(\hat{\varepsilon}) \times 0.4$ in order to get an offset less than 0.4 as follows:

$$
\sum_{n=0}^{2 N-1} y_{1}(n)=\sum_{n=0}^{2 N-1} y_{1}(n) e^{-j 2 \pi S(\hat{\varepsilon}) 0.4 n / N}
$$

After reducing the CFO values to a range less than 0.4, then we will able to estimate the offset by MLE algorithm for less than 0.5 . Consequently, we obtain the precise range less than 0.4 , then add it to the numerical multiplicand of 0.4. Finally, an accurate estimation of CFO values will be obtained as expressed in Eq. (14) :

$$
\widehat{\varepsilon}=\frac{1}{2 \pi} \arg \left\{\sum_{\mathrm{n}=0}^{\mathrm{N}-1} \mathrm{y}_{1}^{*}[\mathrm{n}] \mathrm{y}_{1}[\mathrm{n}+\mathrm{N}]\right\}+0.4 \times \mathrm{S}(\widehat{\varepsilon})
$$

We use 0.4 as multiplicand rather 0.5 because in MLE the maximum range is less than 0.5 , therefore, we can not use a value equal or greater than 0.5. From the Eq. (14) we also can say that when D $=1$, the limit of the acquisition range for the CFO is less than \pm 0.5 and when the acquisition range goes towards the $0.5, \hat{\varepsilon}$ may casue a noise and discontinuity of the arctangent, and it will jump to -0.5 and the estimation become useless. According to the proposed technique we will get a wide range of estimation, which in turn, significantly enhance the performance accuracy.

\section{Performance Analysis}

This section describes the performance analysis of the proposed technique. In the analysis, we assumed that the channel affect is neglected, whereas the noise is considered. MSE has been selected to be the performance metric under simulation tests. The MSE refers to the average error within an OFDM block. The block size of the OFDM symbol has selected to quale to $\mathrm{N}=128$. The simulation tests are carried out at a different signal to noise ratio (SNR), and the length of the guard interval is chosen to be $N / 4=32$. In this analysis, we test the proposed technique and compare it to the the related methods. In the graph of Fig. 1, Moose technique and the proposed technique have the same estimation accuracy, but as in Fig. 2, Moose technique does not work for the CFO greater than 0.5. Fig. 2 also shows that the MSE of D-repetitive decreases when $\mathrm{D}$ increases even with the average of D-repetitive. The proposed technique has better MSE 
performance and less SNR by about $9 \mathrm{~dB}$ to the D-average repetitive and $16 \mathrm{~dB}$ to D-repetitive techniques, that the proposed technique have better estimation result.

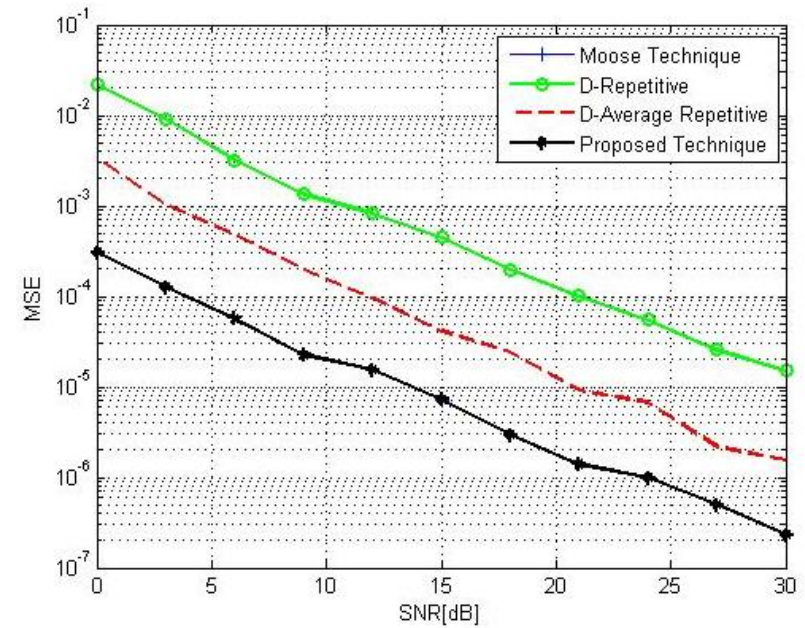

Fig. 1: MSE of CFO estimation $(\mathrm{D}=2, \mathrm{CFO}=0.2)$

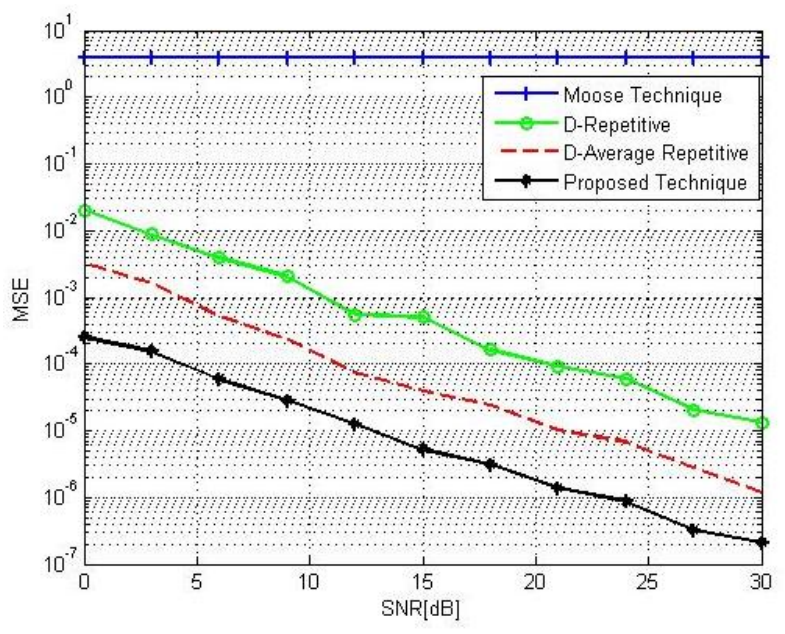

Fig. 2: MSE of CFO estimation $(\mathrm{D}=4, \mathrm{CFO}=1.55)$

\section{Conclusions}

In this paper, we propose a new technique for estimating CFO values in OFDM system. The proposed technique increases the range of estimation with preserve the accuracy. This is accomplished by applying a step function to find a numerical multiplicand of 0.4. Consequently, this reduces the frequency offset in order that MLE algorithm can be applied to values less than 0.5. Various simulation tests have conducted to evaluate and compare the proposed technique to the related methods. The analysis results show that the proposed technique has better accuracy result. As a future work, it will be worth to modify our technique to work with MIMO-OFDM systems.

\section{References}

[1] Guvenkaya, Ertugrul,Self-interference in OFDM-Based Systems: Identification and Separation. 2013.

[2] Simon, E. P., Ros, L., Hijazi, H., \& Ghogho, M. . Joint carrier frequency offset and channel estimation for OFDM systems via the EM algorithm in the presence of very high mobility. IEEE Transactions on Signal Processing. 2012, 60(2): 754-765.

[3] Ibrahim, Shimaa El-sayed, K. A. Elbarbary, and Ragab M. El-Sagheer. Maximizing CFO Estimation Range using a New OFDM Symbol Structure.International Journal of Computer Applications.2014,88(4).

[4] P.H. Moose. A Technique for Orthogonal Frequency Division Multiplexing Frequency Offset Correction. IEEE Transaction on Communications. 1994,42(10): 2908-2914

[5] J.J van de Beek, M. Sandell, and P.O. Borjesson. ML Estimation of Time and Frequency Offset in OFDM Systems. IEEE Transactions on Signal Processing. 1997, 45(7): 1800-1805.

[6] H. Zhou, A.V. Malipatil, and Y.F. Huang.Maximum-likelihood carrier frequency offset estimation for OFDM systems in fading channels. In: Las Vegas, USA.2006,pp. 1461-1464.

[7] F. Classen and H. Meyr.Synchronization algorithms for an OFDM system for mobile communication. ITGFachtagung, 1994: 105-113

[8] Yong Soo Cho, Jaekwon Kim, Won Young Yang, Chung-Gu Kang. MIMO-OFDM Wireless Communication with MATLAB. 2010. 